EPJ Web of Conferences 79, 02001 (2014)

DOI: $10.1051 /$ epjconf/20147902001

(C) Owned by the authors, published by EDP Sciences, 2014

\title{
Thermal conductivity of organic semi-conducting materials using 3omega and photothermal radiometry techniques
}

\author{
Frederic Reisdorffer ${ }^{1}$, Bertrand Garnier ${ }^{2}$, Nicolas Horny ${ }^{3}$, Cedric Renaud ${ }^{4}$, Mihai Chirtoc ${ }^{3}$ \\ and Thien-Phap Nguyen ${ }^{1}$
}

${ }^{1}$ Institut des Matériaux Jean Rouxel, CNRS, University of Nantes, 2 rue de la Houssinière, 44322 Nantes Cedex 3, France

${ }^{2}$ Laboratoire de Thermocinetique, CNRS, University of Nantes, LUNAM, BP. 50609, 44306 Nantes Cedex 3, France

${ }^{3}$ University of Reims Champagne Ardenne URCA, GRESPI, Multiscale Thermophysics Lab., Moulin de la Housse, BP. 1039, 51687 Reims, France

${ }^{4}$ University of Toulouse; UPS, CNRS, INP; Laboratoire Plasma et Conversion d'Energie, Toulouse, France

\begin{abstract}
Organic semiconductors for opto-electronic devices show several defects which can be enhanced while increasing the operating temperature. Their thermal management and especially the reduction of their temperature are of great interest. For the heat transfer study, one has to measure the thermal conductivity of thin film organic materials. However the major difficulty for this measurement is the very low thickness of the films which needs the use of very specific techniques. In our work, the 3-omega and photothermal radiometric methods were used to measure the thermal conductivity of thin film organic semiconducting material (Alq3). The measurements were performed as function of the thin film thickness from 45 to $785 \mathrm{~nm}$ and also of its temperature from 80 to $350 \mathrm{~K}$. With the 3 omega method, a thermal conductivity value of $0.066 \mathrm{~W} \cdot \mathrm{m}^{-1} \mathrm{~K}^{-1}$ was obtained for Alq3 thin film of $200 \mathrm{~nm}$ at room temperature, in close agreement with the photothermal value. Both techniques appear to be complementary: the 3 omega method is easier to implement for large temperature range and small thicknesses down to a few tens of nanometers whereas the photothermal method is more suitable for thicknesses over $200 \mathrm{~nm}$ since it provides additional information such as the thin film volumetric heat capacity.
\end{abstract}

\section{Introduction}

In the global context of energy saving and protection of the eco-system, the interest is growing internationally focused on the development of organic semiconductors for the production of optoelectronic devices at low cost and high performance. Indeed, electrically conductive organic materials are an alternative to inorganic conductors or semi conductors. They are currently used in organic photovoltaic panels [1], organic light emitting diodes (OLEDs) [2] and organic field effect transistors [3]. However, one of their drawbacks is their short lifetime, which is due to premature degradation and

This is an Open Access article distributed under the terms of the Creative Commons Attribution License 4.0, which permits unrestricted use, distribution, and reproduction in any medium, provided the original work is properly cited. 
their weak stability when working at increasing temperature [4]. An example can be found for lighting applications. Compared to other light sources, OLEDs have several advantages such as lightweight, flexibility, large area, wide variety of colors, and are perfectly adapted for building or automotive applications. One of the major drawbacks of organic components is their instabilities during operation, which limits their integration into systems. This instability is due to electrical defects which are enhanced upon increasing temperature. These defects have not been so far well located, identified and analyzed. In light-emitting devices, the power dissipation, which is generally low, may damage the material when the defects are highly localized, particularly at the interfaces of the thin films. It is therefore necessary to understand how energy is dissipated into the devices to increase their stability. It is also important to find ways to decrease the operating temperature to increase their performance in term of brightness and lifetime. Zhou et al. [5] have studied the temperature variation during the polarization of TPD/Alq3 based diodes using infrared thermography and have observed a thermal breakdown, which was responsible for the device failure. The conclusion of this study was the necessity to improve the thermal management of the organic devices. One of the first steps for this task is to know the thermal properties of each material and especially those of the active one. As organic materials are used as thin films (less than $100 \mathrm{~nm}$ ), only very specific thermal conductivity measurement techniques can be used. For small temperature increase (less than $5^{\circ} \mathrm{C}$ ) during the measurement, the two available techniques are the photothermal radiometry (PTR) [6, 7] and the 3omega method (3 $\omega$ ) [8]. In this work, we have used the two previously cited methods to measure the thermal conductivity of 8 tris (hydroxyquinoline) aluminum (Alq3) thin film. This organic semi conducting material is used principally for its lighting properties in OLEDs [2] but is also of great interest for memory application [9]. The thermal conductivity measurements were performed for Alq3 samples of thicknesses from 45 to $785 \mathrm{~nm}$ at room temperature for the PTR method and as function of temperature (from 80 to $350 \mathrm{~K}$ ) for the $3 \omega$ method.

\section{Experimental details}

Alq3 thin films were thermally evaporated on $\mathrm{SiO}_{2}$ or suprasil quartz substrates of thicknesses from 45 to $785 \mathrm{~nm}$ using an evaporation rate between 0.1 and $0.5 \AA . \mathrm{s}^{-1}$. The thickness of the layer was checked and controlled during the deposition process using a quartz balance and measured with a Dektak Veeco 8 profilometer.

For PTR measurement, a $100 \mathrm{~nm}$ thick gold layer was deposited all over the Alq3 film to increase the optical absorption. A $532 \mathrm{~nm}$ laser was used to heat the sample and the beam was modulated with an acousto-optical modulator (Fig. 1). The sample was heated on its surface and infrared thermal waves were collected with two parabolic gold mirrors and focused on $\mathrm{HgCdTe}$ IR detector.

Signal is then preamplified and the amplitude and phase of the emitted thermal wave are measured with a Stanford Research lock-in amplifier which is synchronized with the acousto-optical modulator. More information and details of the PTR measurement technique can be found in [7, 10-14].

For $3 \omega$ measurement, a gold strip with two connecting pads is deposited on Alq3 layer by the sputtering technique (Fig. 2). Typically, the gold strip resistance is about 50-100 $\Omega$ and has a length of $1.4 \mathrm{~mm}$, a thickness of $30 \mathrm{~nm}$ and a width about $30-40 \mu \mathrm{m}(2 \mathrm{~b})$.

The wire characteristics were determined by an optical microscope calibrated with sub micrometer standard and by a Dektak Veeco 8 profilometer. Measurements were performed using a Tektronix AGF 3022B function generator as an ac voltage source of the Wheatstone bridge. Current and voltage at the resistance were measured for each frequency using a Keithley 199 and a Keithley 2000 multimeters. The $3 \omega$ detection was performed using a 7265 Ametek lock-in amplifier. The measurement principle is standard for this type of measurements and more information can be found in [8]. To study the effect of temperature on thermal conductivity of Alq3, the samples were placed inside an Ice Oxford cryostat working under a pressure of $\sim 10^{-5}$ mbar and temperature was controlled in the range 80-360 K 


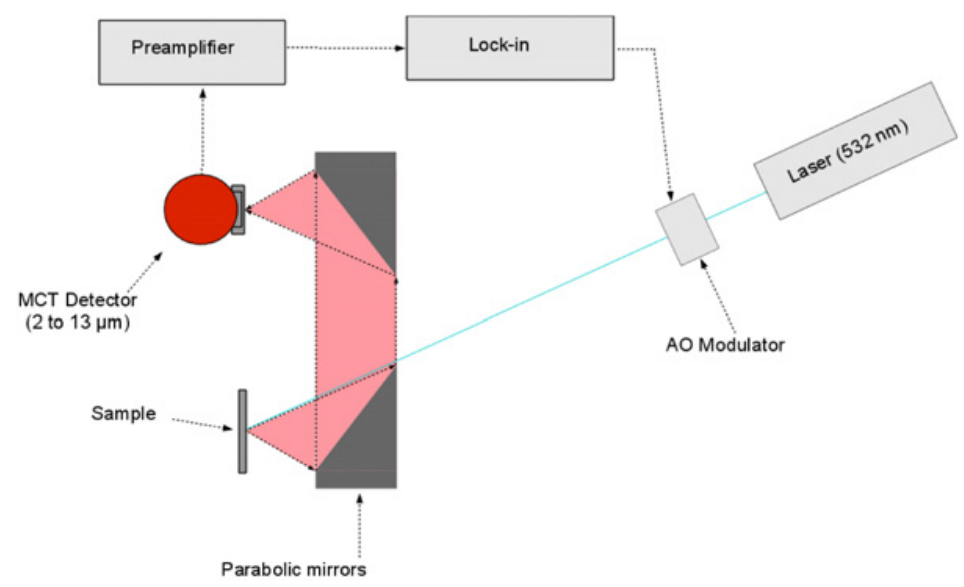

Figure 1. Experimental setup for the photothermal radiometry technique (PTR) [7].

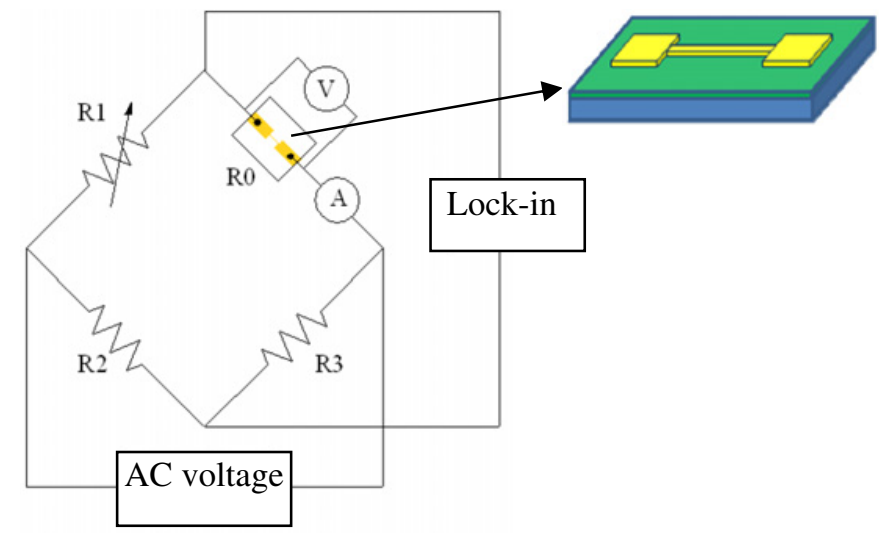

Figure 2. Experimental setup for the 3omega method $(3 \omega)$.

with a Cry-con 32B temperature controller. A systematic protocol was repeated for each temperature to measure the Wheatstone bridge unbalanced voltage. Thus when the set point temperature was reached, the Wheatstone bridge was equilibrated and all its resistances were measured. Then a frequency scan with 150 values was performed from a high frequency of about $10 \mathrm{kHz}$ down to $1 \mathrm{~Hz}$ while the lockin integration time was adjusted in the range from 1 to $5 \mathrm{~s}$ depending on the frequency value. All the experimental procedure was automated using Labview software.

\section{Results and discussion}

Photothermal radiometric measurements are reported in Fig. 3 showing a typical Bode diagram of a) the amplitude-square root of the frequency product versus frequency and b) the phase versus frequency for different thicknesses of the Alq3 layer. Amplitude and phase are normalized using those obtained with the gold layer only on same substrate to estimate the transfer function. The obtained quantities as shown in Fig. 3 depend strongly on the Alq3 thin film thickness. Indeed, for increasing Alq3 thickness, a shift towards lower frequency is observed on the amplitude and phase diagrams. This indicates that the time to cross the layer increases with the layer thickness. It is now necessary to see if these variations are due 

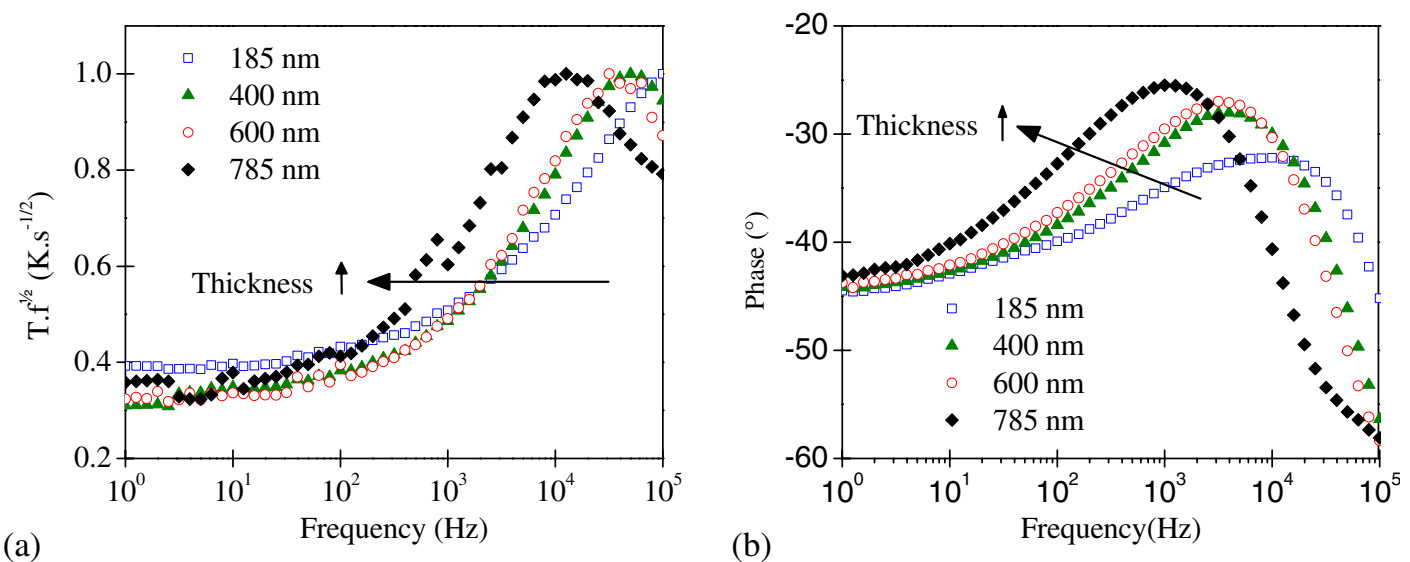

Figure 3. Photothermal measurements for different thicknesses of Alq 3 thin films: a) normalized product of the amplitude and of the square root of the frequency versus frequency and b) phase versus frequency.
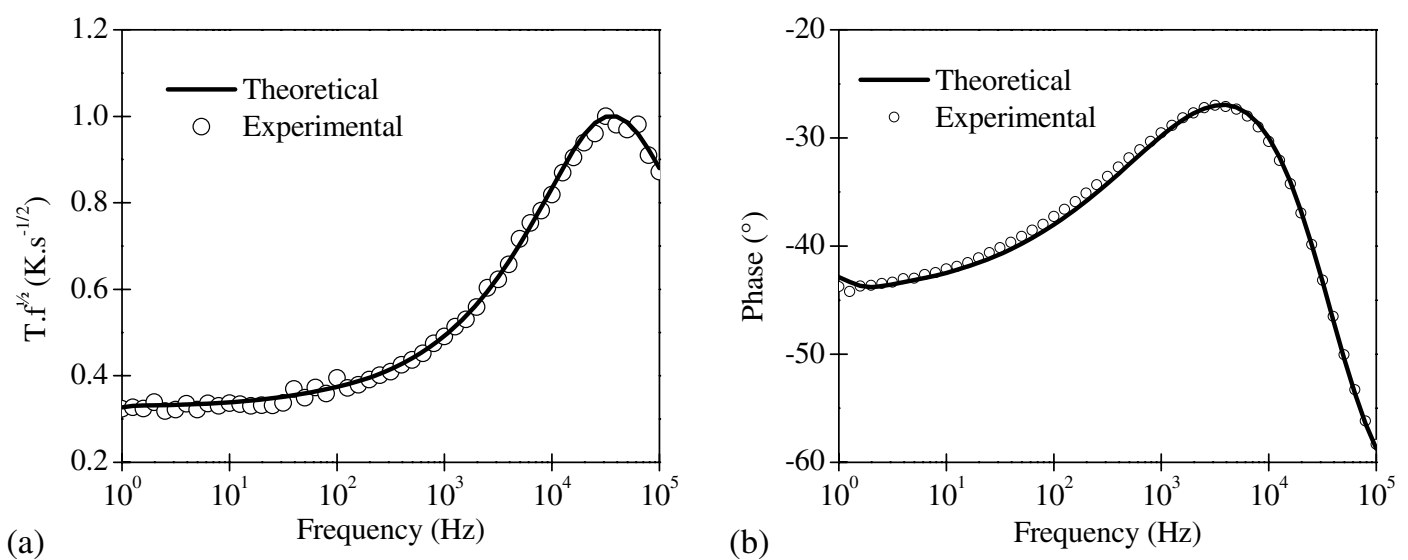

Figure 4. Superposition of PTR measurements and of the theoretical curves for a $600 \mathrm{~nm}$ thick Alq3 layer: a) normalized product of the amplitude and of the square root of the frequency versus frequency and b) phase versus frequency.

only to the thicknesses or also to variations of thermal properties of the film. To obtain the Alq3 thermal conductivity, a theoretical thermal model is necessary. A 1D heat transfer model, using three layers, was established by neglecting all the interfacial thermal resistances and with a front detection configuration. This has been described by several previous works [7, 10, 13, 15, 16].

In this model, a thermal conductivity of $1.2 \mathrm{~W} \cdot \mathrm{m}^{-1} \cdot \mathrm{K}^{-1}$, a specific heat of $800 \mathrm{~J} \cdot \mathrm{kg}^{-1} \cdot \mathrm{K}^{-1}$ and a density of $2490 \mathrm{~kg} \cdot \mathrm{m}^{-3}$ were considered for the $1 \mathrm{~mm}$ thick glass substrate. Concerning the $100 \mathrm{~nm}$ thick gold layer, a thermal conductivity of $317 \mathrm{~W} \cdot \mathrm{m}^{-1} \cdot \mathrm{K}^{-1}$, a specific heat of $189 \mathrm{~J} \cdot \mathrm{kg}^{-1} \cdot \mathrm{K}^{-1}$ and a density of $19300 \mathrm{~kg} \cdot \mathrm{m}^{-3}$ were considered.

Figure 4 shows the experimental and theoretical results obtained for a $600 \mathrm{~nm}$ thick Alq3 layer. It can be seen that the signal amplitude and phase are well described by the model used. The thermal conductivity of Alq3 layer is about $0.05-0.08 \mathrm{~W} \cdot \mathrm{m}^{-1} \cdot \mathrm{K}^{-1}$ for thicknesses in the range 185 to $785 \mathrm{~nm}$. The results indicate noticeable thickness dependence and the model used cannot totally explain the phase curve for the $185 \mathrm{~nm}$ thick Alq3 layer, which indicates that the Alq3 properties are probably different for 
$3^{\text {rd }}$ European Energy Conference

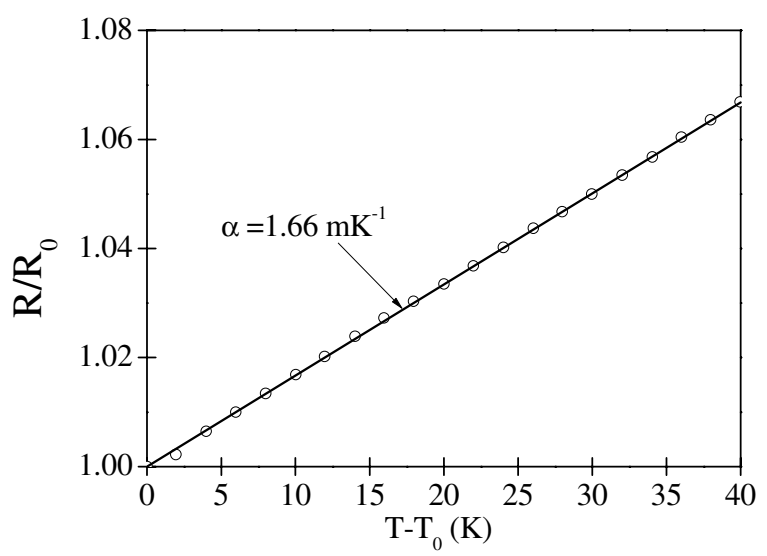

Figure 5. Measurement of the temperature coefficient of the gold strip electrical resistance.

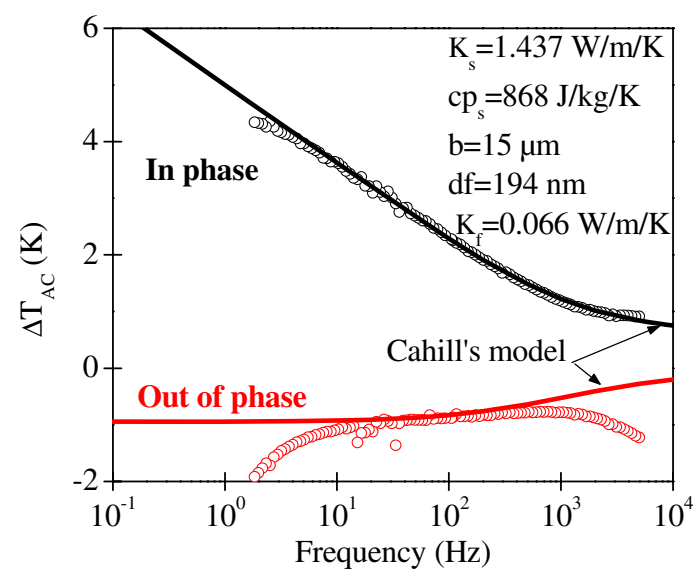

Figure 6. Thermal oscillations at $300 \mathrm{~K}$ using $3 \omega$ method: superposition of real (in phase) and imaginary part (out of phase) of the measured thermal oscillations and Cahill's model.

layers of small thicknesses. However, agreement between the $\mathrm{Tf}^{1 / 2}$ theoretical model and experimental results is sufficient to suggest that the thermal conductivity determinations are correct also for this range of thicknesses. With this method, the $\rho$ Cp product for Alq 3 was also estimated and was found to be 2-3.5 $10^{6} \mathrm{~J} \cdot \mathrm{m}^{-1} \cdot \mathrm{K}^{-1}$.

Besides, $3 \omega$ measurements on the same material were also performed. For this method, we have used a typical two pads technique connected to a gold strip. The first step in this method consists of determining the temperature coefficient of the electrical resistance of the gold strip.

For this purpose, we have measured its electrical resistance from 298 to $340 \mathrm{~K}$ with a temperature step of $2 \mathrm{~K}$. The temperature coefficient for the electrical resistance of the gold strip determined from figure 5 is $1.66 \mathrm{mK}^{-1}$. Accurate determination of this coefficient is essential to measure the thermal conductivity. Indeed, during the conversion of the Wheatstone bridge unbalance voltage into temperature oscillations, it is necessary to use this parameter.

In Fig. 6, we have reported the Bode diagram of the real and imaginary parts of the thermal oscillations. In this figure, the corresponding plots from Cahill's model $[8,17-21]$ are also shown. We 

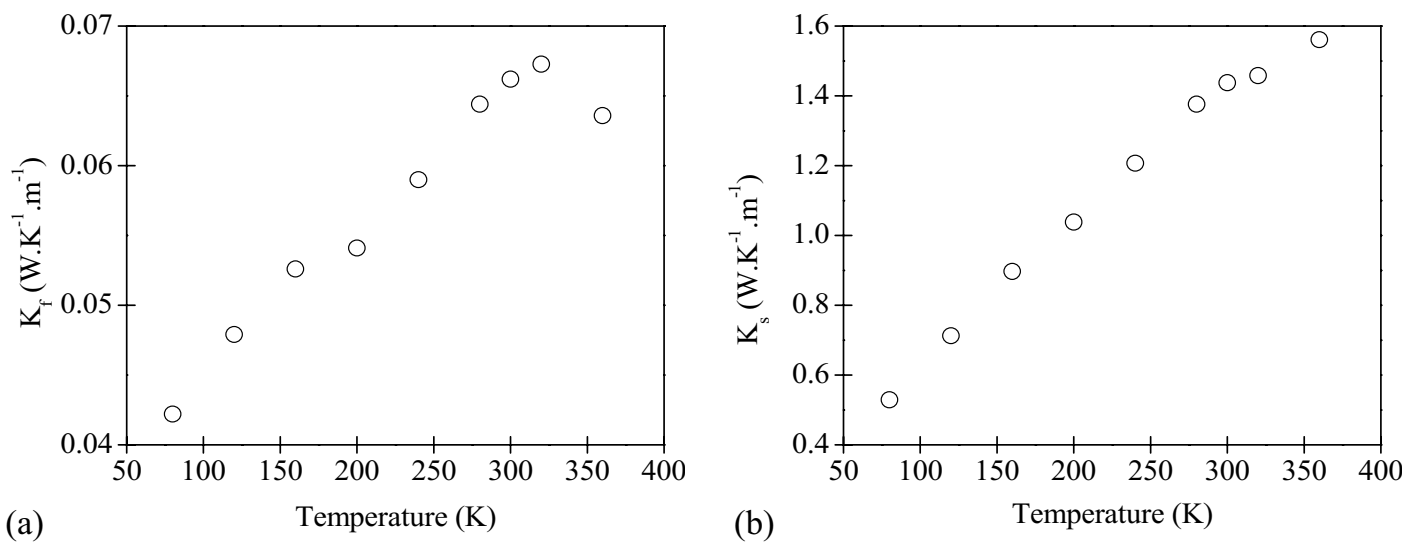

(c)

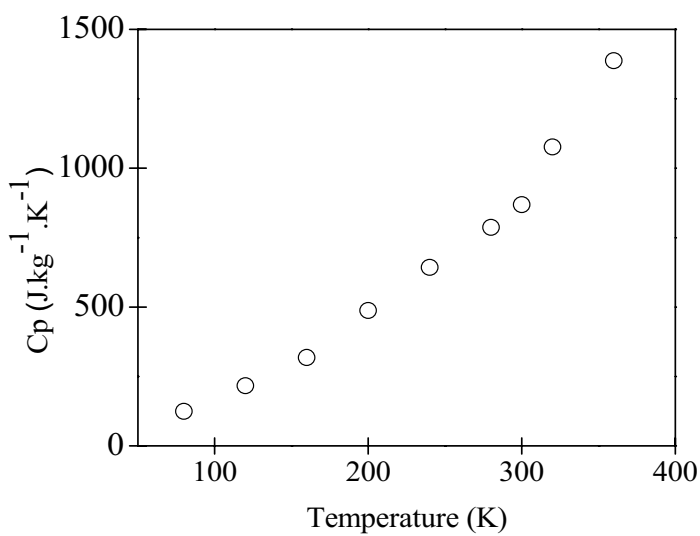

Figure 7. Measured thermal properties obtained with Cahill's $3 \omega$ model as function of the temperature: a) thermal conductivity of Alq3 thin film, b) thermal conductivity of the substrate (suprasil quartz) and c) specific heat of the substrate (suprasil quartz).

note a fair agreement between the experimental and theoretical curves, except for the high frequencies where a deviation of both real and imaginary parts from the calculated plots are observed. This may originate from the capacitive effect of the connecting wires [22]. This will affect the specific heat value (planar regime) [23] but does not change the linear part used to determined the thermal conductivity. We have performed integral calculations using Simpson quadrature combined with simplex or non linear parameter estimation methods to find thermal properties of Alq3 thin film and substrate. We did not use the differential method to determine the thermal conductivity of the thin film because of a problem of parallelism between in phase diagrams obtained with and without the film. This issue is probably linked to the nature of interface, which is not the same in both cases. However, using Cahill's model, we obtained a good agreement between measured and theoretical in-phase and out-of-phase diagrams (Fig. 6). In addition, the thermal characteristics determined by us are in agreement with those reported in the literature. Indeed, Figs. 7a and 7c show results obtained on quartz suprasil substrate and we have found that its thermal conductivity is about 1.38 and $144 \mathrm{~W} \cdot \mathrm{m}^{-1} \cdot \mathrm{K}^{-1}$ at respectively 280 and $300 \mathrm{~K}$ with the following specific heat of 785 and $868 \mathrm{~J} \cdot \mathrm{kg}^{-1} \cdot \mathrm{K}^{-1}$. It is very close to the values previously determined for this material at comparable temperature range [24]. Consequently, the experimental procedure and calibration of our experiments are suitable for measuring the substrate thermal properties. 


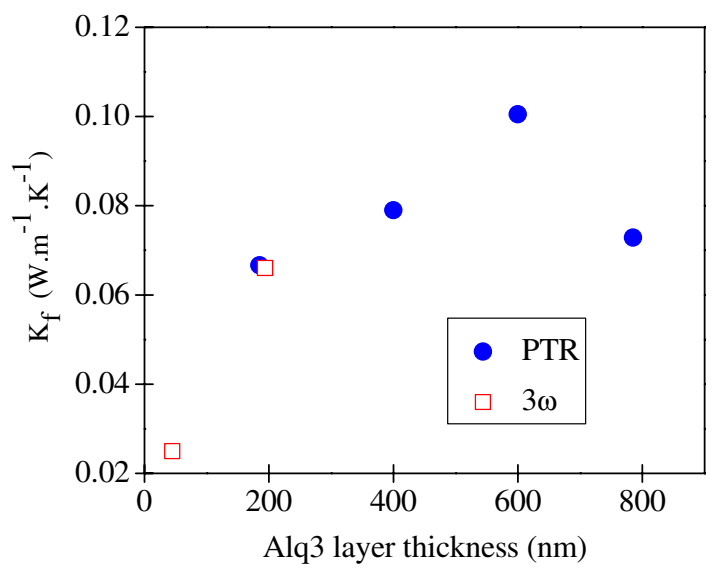

Figure 8. Variations of the Alq3 thermal conductivity versus sample thicknesses as measured by the PTR and $3 \omega$ methods.

We have determined the thermal conductivity of Alq3 thin films of 45 and $194 \mathrm{~nm}$ thick and we show on the Fig. $7 \mathrm{~b}$ data obtained for the $194 \mathrm{~nm}$ thin film. We can see that the measured Alq3 thermal conductivity at $300 \mathrm{~K}$ is about $0.067 \mathrm{~W} \cdot \mathrm{m}^{-1} \cdot \mathrm{K}^{-1}$ with a standard error of about $0.01 \mathrm{~W} \cdot \mathrm{m}^{-1} \cdot \mathrm{K}^{-1}$. Indeed, for frequencies less than $5 \mathrm{~Hz}$, we can observe a deviation due to the thermal wave reaching the back side of the substrate and penetrating into the cryostat metallic sample holder. Another source of disturbance is the electrical network, which affects the frequency scan, especially around 50 and $100 \mathrm{~Hz}$ and causes strong artefacts.

The values found for the Alq3 thermal conductivity are smaller than those obtained in literature. Indeed, Shin et al. [25] has obtained a thermal conductivity of $0.107 \mathrm{~W} \cdot \mathrm{m}^{-1} \cdot \mathrm{K}^{-1}$ on Alq3 pellet. In comparison, our values are of the same order of magnitude.

The discrepancy may be due to the fact that the measurements were performed on different Alq3 samples, the structure of which may vary and depends on the deposition conditions. In another study, Kim et al. [26] have found a thermal conductivity of $0.5 \mathrm{~W} \cdot \mathrm{m}^{-1} \cdot \mathrm{K}^{-1}$ for thin film of thickness from 0.2 to $1 \mu \mathrm{m}$ using $3 \omega$ methods with a similar experimental protocol. However, the principal difference is on their fabrication process. Indeed, they have sublimated their powder before the deposition of Alq3 thin films. Consequently, their films are purified by comparison to ours, and this can explain the higher thermal conductivity value. Therefore, the organization of the layer is probably totally different and it shows that it is possible to tune the thermal properties by changing the deposition or purification process.

Figure 8 shows the superposition of the various thermal conductivities that we have measured by the PTR and $3 \omega$ methods at room temperature. First, we can see a close agreement between the two methods. Indeed, a thermal conductivity about $0.067 \mathrm{~W} \cdot \mathrm{m}^{-1} \cdot \mathrm{K}^{-1}$ was found by the PTR method for a Alq3 thin film of $185 \mathrm{~nm}$ and a thermal conductivity of $0.066 \mathrm{~W} \cdot \mathrm{m}^{-1} \cdot \mathrm{K}^{-1}$ was obtained with the $3 \omega$ method for an Alq3 thin film of $194 \mathrm{~nm}$. On the other hand, the data obtained by PTR and those obtained by $3 \omega$ appears to complete each other in term of range of thin film thicknesses. Moreover, we have determined a thermal conductivity of $0.025 \mathrm{~W} \cdot \mathrm{m}^{-1} \cdot \mathrm{K}^{-1}$ for an Alq3 thin film of $45 \mathrm{~nm}$. It should be noted that such a small thickness has never been used in thermal conductivity measurements. This result indicates a thickness dependence of the Alq3 thermal conductivity, which decreases when the film thickness decreases.

Currently, we are studying the degradation process of Alq3 thin films and we have observed the onset of a novel component for thicknesses less than $400 \mathrm{~nm}$. This new component is visible only before degradation of the $185 \mathrm{~nm}$ film. Consequently, there might be formation of a new species in the film 
with different thermal properties during Alq3 thin film aging. Further studies are necessary to better investigate the thermal contribution in thin film aging of OLED active layer which contributes to their degradation process.

\section{Conclusions}

Two different methods (PTR and $3 \omega$ ) were used to measure the thermal conductivity of Alq3 thin films. A close agreement between the results obtained by both techniques was found. For a temperature of $300 \mathrm{~K}$, a thermal conductivity about $0.067 \mathrm{~W} \cdot \mathrm{m}^{-1} \cdot \mathrm{K}^{-1}$ was obtained for $200 \mathrm{~nm}$ thick Alq3 thin films. This study has shown the importance to use several characterization techniques to determine thermal properties of organic layers in OLEDs since the thermal measurements are difficult with samples of thickness less than $1 \mu \mathrm{m}$. In addition, we have shown that the thermal conductivity is dependent on the thickness of the layer, which is about $45 \mathrm{~nm}$ in OLED application. Indeed, the thermal conductivity of Alq3 tends to decrease for thicknesses below $200 \mathrm{~nm}$ probably due to a different organization of the layer at this scale. In the future, these thermal conductivity values will be used in thermal management models to try to reduce the operating temperature of OLED using a passive or active cooling device.

\section{References}

[1] C.W. Tang, Appl. Phys. Lett. 48 183-185 (1986)

[2] C. Tang, S. VanSlyke, Appl. Phys. Lett. 51 913-915 (1987)

[3] H. Koezuka, A. Tsumura, T. Ando, Synth. Met. 18 699-704 (1987)

[4] F. So, D. Kondakov, Adv. Mater. 22 3762-3777 (2010)

[5] X. Zhou, J. He, L.S. Liao, M. Lu, X.M. Ding, X.Y. Hou, et al., Adv. Mater. 12 265-269 (2000)

[6] M.E. Rodrı̀guez, J.A. Garcia, A. Mandelis, Rev. Sci. Instrum. 74 839-841 (2003)

[7] M. Chirtoc, N. Horny, I. Tavman, A. Turgut, I. Kökey, M. Omastová, Int. J. Therm. Sci. 62 50-55 (2012)

[8] D.G. Cahill, Rev. Sci. Instrum. 61 802-808 (1990)

[9] Chia-Hsun Tu, Yi-Sheng Lai, Dim-Lee Kwong, IEEE Electron Device Lett. 27 354-356 (2006)

[10] M. Chirtoc, J. Phys. Conf. Ser. 214012005 (2010)

[11] M. Chirtoc, E.H. Bentefour, C. Glorieux, J. Thoen, Thermochim. Acta. 377 105-112 (2001)

[12] A. Gijsbertsen, D. Bicanic, J.L.. Gielen, M. Chirtoc, Infrared Phys. Technol. 45 93-101 (2004)

[13] N. Horny, M. Chirtoc, Y. Kanaké, L. Tighzert, CR Rom Proc. Congrès Société Française Thermique, Géradmer, France, May 28-31 (2013)

[14] P. Kijamnajsuk, J. Pelzl, M. Chirtoc, N. Horny, D. Schäfer, C. Eisenmenger-Sittner, Int. J. Thermophys. 33 2132-2138 (2012)

[15] M. Chirtoc, Investigation of layered systems by photothermal methods with periodic excitation, in: Thermal Wave Physics Related Photothermal Techniques: Basic Principles and Recent Developments, pp. 29-63, ed. E. Marín Moares, Transworld Research Network, Kerala, India 2009

[16] Ph. Grossel, F. Depasse, Int. J. Thermal Sci., 50(11) 2078-2083 (2011)

[17] D.G. Cahill, W.K. Ford, K.E. Goodson, G.D. Mahan, A. Majumdar, H.J. Maris et al., J. Appl. Phys. 93054303 (2003)

[18] S. Ahmed, R. Liske, T. Wunderer, M. Leonhardt, R. Ziervogel, C. Fansler, et al., Diam. Relat. Mater.15 389-393 (2006)

[19] N. Al-Khudary, P.Y. Cresson, T. Lasri, Journées Nationales du Réseau Doctoral en Micronanoélectronique, Marseille France (2012) JNRDM 2012

[20] T. Yamane, N. Nagai, S. Katayama, M. Todoki, J. Appl. Phys. $919772-9776$ (2002)

[21] N.O. Birge, S.R. Nagel, Rev. Sci. Instrum. 58 1464-1470 (1987) 
$3^{\text {rd }}$ European Energy Conference

[22] N.O. Birge, P.K. Dixon, N. Menon, Thermochim. Acta. 304 51-66 (1997)

[23] I.K. Moon, Y.H. Jeong, S. Kwun, Rev. Sci. Instrum. 67 29-35 (1996)

[24] D.H. Damon, Phys. Rev. B. 8 5860-5865 (1973)

[25] M. Whan Shin, H. Chul Lee, K. Soo Kim, S.-H. Lee, J.-C. Kim, Thin Solid Films 363 244-247 (2000)

[26] N. Kim, B. Domercq, S. Yoo, A. Christensen, B. Kippelen, S. Graham, Appl. Phys. Lett.87 241908 (2005) 